\title{
Daprodustat Compared with Epoetin Beta Pegol for Anemia in Japanese Patients Not on Dialysis: A 52-Week Randomized Open-Label Phase 3 Trial
}

\author{
Masaomi Nangaku ${ }^{a}$ Takayuki Hamano ${ }^{b}$ Tadao Akizawa $^{c}$

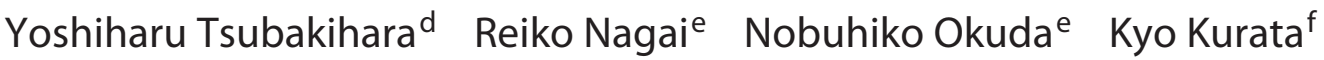 \\ Takashi Nagakubo $^{g}$ Nigel P. Jones ${ }^{\mathrm{h}} \quad$ Yukihiro Endo $^{\mathrm{e}} \quad$ Alexander R. Cobitz $^{\mathrm{i}}$ \\ aDivision of Nephrology and Endocrinology, The University of Tokyo, Graduate School of Medicine, Tokyo, Japan; \\ ${ }^{b}$ Department of Nephrology, Nagoya City University Graduate School of Medical Sciences, Aichi, Japan; 'Division \\ of Nephrology, Department of Medicine, Showa University School of Medicine, Tokyo, Japan; 'Graduate School \\ of Health Care Sciences, Jikei Institute, Osaka, Japan; 'Medicines Development, GlaxoSmithKline, Tokyo, Japan; \\ fMedical Affairs \& Development, GlaxoSmithKline, Tokyo, Japan; 'BBiomedical Data Sciences, GlaxoSmithKline, \\ Tokyo, Japan; ${ }^{h}$ Clinical Sciences, GlaxoSmithKline, Uxbridge, UK; i Clinical Sciences, GlaxoSmithKline, Collegeville, PA, \\ USA
}

\section{Keywords}

Anemia · Daprodustat · Hypoxia-inducible factor · Japanese · Nondialysis

\begin{abstract}
Background: Daprodustat is an oral agent that stimulates erythropoiesis by inhibiting the prolyl hydroxylases which mark hypoxia-inducible factor for degradation through hydroxylation. Its safety and efficacy (noninferiority) were assessed in this 52-week, open-label study. Methods: Japanese patients not on dialysis (ND) $(N=299)$ with anemia of CKD (stages G3, G4, and G5) with iron parameters of ferritin $>100$ $\mathrm{ng} / \mathrm{mL}$ or transferrin saturation $>20 \%$ at screening were randomized to daprodustat or epoetin beta pegol (continuous erythropoietin receptor activator [CERA], also known as methoxy polyethylene glycol-epoetin beta). After initiation of the study, the daprodustat starting dose for erythropoiesisstimulating agent (ESA)-naïve participants was revised, and daprodustat was started at 2 or $4 \mathrm{mg}$ once daily depending on baseline hemoglobin. ESA users switched to daprodustat
\end{abstract}

karger@karger.com www.karger.com/ajn

Karger $\stackrel{\text { ' }}{5}$

GOPEN ACCESS
(C) 2021 The Author(s)

Published by S. Karger AG, Basel

This is an Open Access article licensed under the Creative Commons Attribution-NonCommercial-4.0 International License (CC BY-NC) (http://www.karger.com/Services/OpenAccessLicense), applicable to the online version of the article only. Usage and distribution for commercial purposes requires written permission.
$4 \mathrm{mg}$ once daily. CERA was started at $25 \mu \mathrm{g}$ every 2 weeks for ESA-naïve patients and 25-250 $\mu \mathrm{g}$ every 4 weeks for ESA users based on previous ESA dose. In both treatment groups, dose was adjusted every 4 weeks based on hemoglobin level and changed according to a prespecified algorithm. The primary end point was mean hemoglobin level during weeks 40-52 in the intention-to-treat (ITT) population. ESA-naïve patients who entered before the protocol amendment revising the daprodustat starting dose were excluded from the ITT population. Results: Mean hemoglobin levels during weeks $40-52$ were $12.0 \mathrm{~g} / \mathrm{dL}$ in the daprodustat group ( $n=$ $108 ; 95 \%$ confidence interval [Cl], 11.8-12.1) and $11.9 \mathrm{~g} / \mathrm{dL}$ for CERA ( $n=109 ; 95 \% \mathrm{Cl} 11.7-12.0)$; the difference between the groups was $0.1 \mathrm{~g} / \mathrm{dL}(95 \% \mathrm{Cl}-0.1$ to $0.3 \mathrm{~g} / \mathrm{dL})$. The lower limit of the $95 \% \mathrm{Cl}$ of the difference was greater than the prespecified margin of $-1.0 \mathrm{~g} / \mathrm{dL}$. The mean hemoglobin level was within the target range $(11.0-13.0 \mathrm{~g} / \mathrm{dL})$ during weeks $40-52$ for $92 \%$ of participants in both groups. There was no meaningful difference in the frequencies of adverse events.

ClinicalTrials.gov Identifier: NCT02791763. 
Conclusions: Oral daprodustat was noninferior to CERA in achieving and maintaining target hemoglobin levels in Japanese ND patients. Daprodustat was well tolerated, with no new safety concerns identified. @ 2021 The Author(s)

Published by S. Karger AG, Basel

\section{Introduction}

Anemia is a common complication of CKD [1] caused by relative EPO insufficiency [2]. Erythropoiesis-stimulating agents (ESAs) and concomitant iron supplementation are the standard of care for treatment of anemia of CKD in Japan [2]. However, ESAs have been associated with an increased risk of cardiovascular events (stroke, myocardial infarction, and all-cause mortality) and exacerbation of hypertension when treating to higher hemoglobin targets than recommended [3-8]. It is unclear if the risk is related to the rapid hemoglobin rise or the dose of ESA used.

Daprodustat is a hypoxia-inducible factor (HIF)-prolyl hydroxylase inhibitor (PHI), an emerging class of therapy for anemia of CKD [9-13]. Inhibition of HIF-prolyl hydroxylase leads to activation of HIF-responsive genes that stimulate erythropoiesis. Additionally, HIF activation induces genes involved in iron uptake, mobilization, and transport including transferrin and decreases hepcidin by indirect mechanisms [10]. In phase 2 studies, daprodustat $0.5-25 \mathrm{mg}$ once daily achieved and maintained target hemoglobin levels in hemodialysis patients and patients not on dialysis (ND) $[9,14,15]$.

In this phase 3 study, we tested the hypothesis that daprodustat is noninferior to epoetin beta pegol (continuous erythropoietin receptor activator [CERA], also known as methoxy polyethylene glycol-epoetin beta) in maintaining hemoglobin levels in Japanese ND patients with anemia of CKD. The 1-year safety of daprodustat was also evaluated.

\section{Materials and Methods}

\section{Study Design}

This phase 3, open-label, active-controlled, parallel-group study was conducted in Japanese ND patients with anemia of CKD (NCT02791763). Participants from 56 sites were entered between June 2016 and October 2018. After a 4-week screening period, 299 patients (117 ESA users and 182 ESA-naïve) were randomized 1:1 to receive daprodustat or CERA for 52 weeks.

A biostatistician generated the randomization codes using a company-validated system. A random permutation of treatment assignments within blocks (block size was set to 4) stratified by current ESA use/nonuse and the hemoglobin level (ESA-naïve: $\leq 9.5 \mathrm{~g} / \mathrm{dL},>9.5 \mathrm{~g} / \mathrm{dL}$, ESA users: $<11.0 \mathrm{~g} / \mathrm{dL}, \geq 11.0 \mathrm{~g} / \mathrm{dL}$ ) was used for the randomization sequence. Investigators accessed the Interactive Web Response System to obtain the assigned interventions. For both treatment groups, the target hemoglobin range (11.0$13.0 \mathrm{~g} / \mathrm{dL}$ ) was based on Japanese Society for Dialysis Therapy guidelines [16].

The study was approved by the institutional review board (see online suppl. Table 1; for all online suppl. material, see www. karger.com/doi/10.1159/000513103) at every participating institution and was conducted according to the recommendations of Good Clinical Practice and the Declaration of Helsinki. All participants provided written, informed consent to participate in the study.

\section{Participants}

Adults with CKD stages G3, G4, and G5, ND in the previous 12 weeks, with hemoglobin $\geq 8.0 \mathrm{~g} / \mathrm{dL}$ and $<11.0 \mathrm{~g} / \mathrm{dL}$ (ESA-naïve) or $\geq 9.0 \mathrm{~g} / \mathrm{dL}$ and $\leq 13.0 \mathrm{~g} / \mathrm{dL}$ (ESA users) were eligible (online suppl. Table 2). Ferritin $>100 \mathrm{ng} / \mathrm{mL}$ or transferrin saturation (TSAT) $>20 \%$ for both groups was required.

Supplemental iron therapy was to be used if ferritin was $\leq 100$ $\mathrm{ng} / \mathrm{mL}$ and TSAT $\leq 20 \%$; intravenous iron was not allowed from screening through week 4 . Restrictions for iron-containing phosphate binders are shown in online suppl. Table 2. Commercially available ESA use was prohibited during study treatment and for ESA-naïve participants 8 weeks before screening.

\section{Study Assessments and Procedures}

Initially, daprodustat was started at $4 \mathrm{mg}$ once daily in ESAnaïve patients. An ad hoc blinded review of week 4 data showed 8 of 62 (13\%; 6 participants in the daprodustat group, 2 in the CERA group from post hoc analyses) ESA-naïve participants had a hemoglobin increase of $>2.0 \mathrm{~g} / \mathrm{dL}$ from baseline. Consequently, the protocol was amended to lower the daprodustat starting dose from 4 to $2 \mathrm{mg}$ for newly enrolled ESA-naïve patients with a baseline hemoglobin level of $\geq 9.0 \mathrm{~g} / \mathrm{dL}$. All ESA users started daprodustat at $4 \mathrm{mg}$ (before and after the protocol amendment). From week 4 onward, daprodustat dose was titrated every 4 weeks within the range of 1-24 mg according to a prespecified dose adjustment algorithm (online suppl. Table 3).

CERA was started at $25 \mu \mathrm{g}$ every 2 weeks for ESA-naïve patients and 25-250 $\mu$ g every 4 weeks for ESA users (online suppl. Table 4). Dose interval change and dose replacement conversions were made according to a prespecified algorithm. From week 4 onward, dose adjustments were made every 4 weeks according to a prespecified algorithm (online suppl. Table 5).

When the starting dose was amended, a week 2 visit was added to monitor hemoglobin levels regardless of the treatment group. When hemoglobin increased $>1.0 \mathrm{~g} / \mathrm{dL}$ over 2 weeks, the dose of daprodustat or CERA was to be reduced by 1 dose step.

Hemoglobin was measured every 4 weeks. A central laboratory (LSI Medience Corporation; Tokyo, Japan) was used for efficacy assessments, and HemoCue (a point-of-care hemoglobin analyzer) was used for eligibility, withdrawal, and dose adjustment criteria.

Safety assessments included incidence of adverse events (AEs) and AEs of special interest. AEs of special interest were defined a priori based on findings of nonclinical studies of daprodustat, theoretical or potential risks based on the mechanism of action of daprodustat, and the known safety profile of ESAs. A Safety Review 


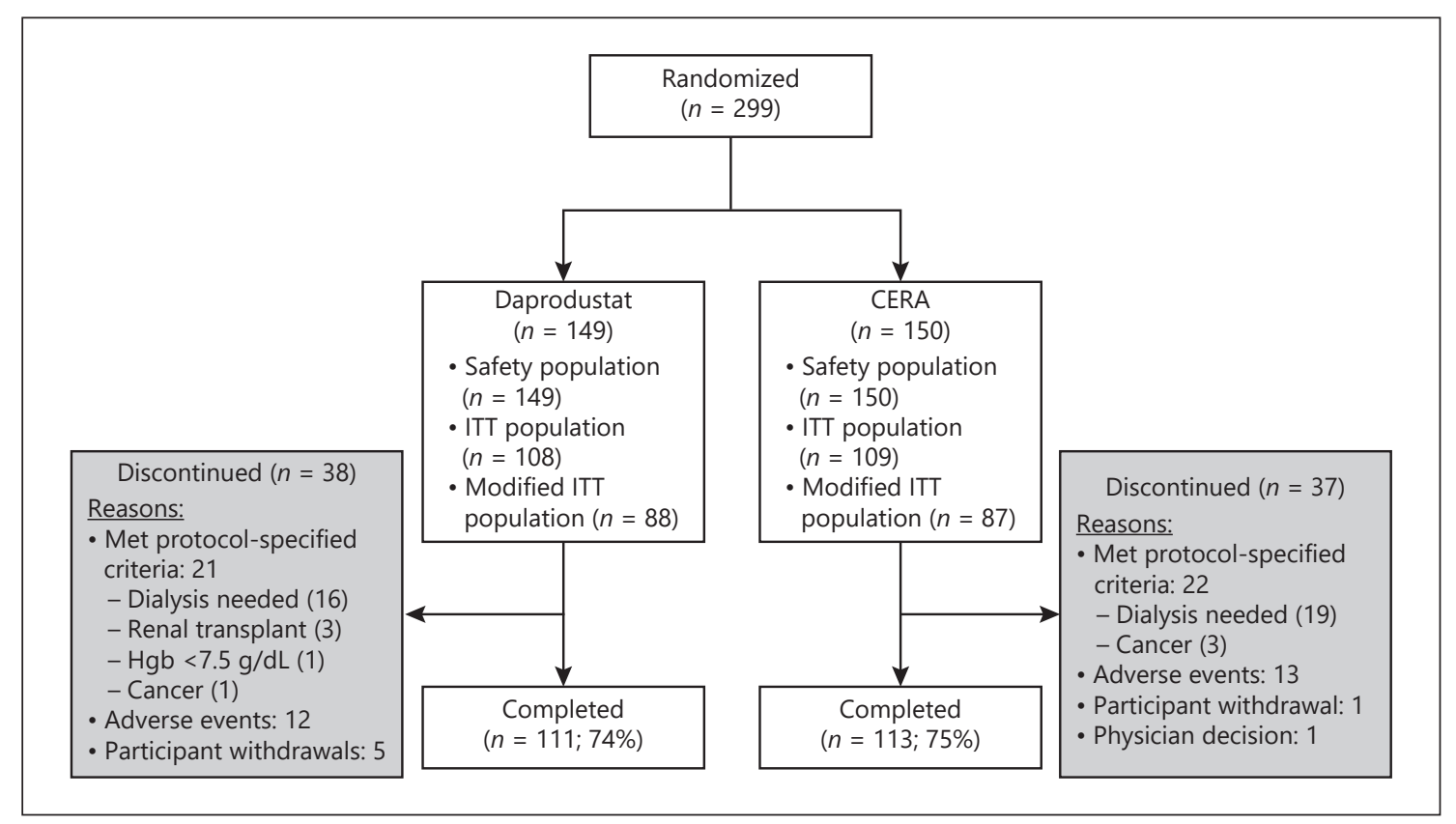

Fig. 1. Participant disposition. This study also evaluated efficacy and safety of daprodustat in a noncomparative cohort of Japanese peritoneal dialysis patients (ClinicalTrials.gov Identifier: NCT02791763). A total of 455 patients (ND and peritoneal dialysis) were screened. Of the 455 screened patients, 355 (78\%) were enrolled. Of the 355 enrolled participants in this study, 299 were ND and 56 were on peritoneal dialysis. CERA, continuous erythropoietin receptor activator (epoetin beta pegol); Hgb, hemoglobin; ITT, intent-to-treat; ND, not on dialysis.

Team blinded to treatment assignment conducted periodic case reviews to evaluate which events constituted AEs of special interest. Protocol-specific ophthalmology examinations were performed by an unblinded ophthalmology specialist for each site at baseline and weeks 12 and 48 for the assessment of ocular AEs of special interest.

\section{Trial Endpoints}

The primary study endpoint was mean hemoglobin concentration during weeks $40-52$ (primary efficacy evaluation period). The principal secondary endpoint was number (\%) of participants with mean hemoglobin levels in the target range during that period. Other secondary endpoints included hemoglobin and hemoglobin change from baseline at each assessment time-point, hemoglobin change from baseline to week 4, time in hemoglobin target range during the primary efficacy evaluation period, daprodustat dose, iron parameters, change from baseline in lipids, incidence of AEs and AEs of special interest, and vital signs.

\section{Statistical Analyses}

Assuming a treatment difference of $0.0 \mathrm{~g} / \mathrm{dL}$, and a SD of $1.5 \mathrm{~g} /$ $\mathrm{dL}$ for the primary endpoint, the noninferiority test had at least $99 \%$ power at a one-sided significance level of $2.5 \%$ with a sample size of 100 participants per group and a noninferiority margin of $-1.0 \mathrm{~g} / \mathrm{dL}$ at study initiation. Anticipating a dropout rate of $30 \%$, 143 participants per group were to be randomized. The study was also designed to ensure long-term safety data from 100 participants in the daprodustat group.
The intention-to-treat (ITT) population included participants with baseline and $\geq 1$ post-baseline hemoglobin, excluding ESAnaïve participants who received treatment prior to the protocol amendment revising daprodustat starting dose. The ITT population was the primary population for noninferiority assessment. Due to the protocol amendment, 82 participants were excluded from the ITT population. Assuming a dropout rate of 30\%, 70 participants per group were anticipated for the noninferiority assessment, and the power was determined to be at least $97 \%$. The modified ITT population consisted of ITT participants with $\geq 1$ hemoglobin during weeks $40-52$. The safety population included all participants who received $\geq 1$ dose of study medication.

Mixed model for repeated measures was used to estimate mean hemoglobin during the primary efficacy evaluation period. The model included treatment groups, baseline hemoglobin, assessment visits, interaction between treatment groups and assessment visits, and interaction between baseline hemoglobin and assessment visits. The hypothesis that daprodustat was noninferior to CERA was to be established if the lower limit of the $95 \%$ confidence interval (CI) was $\geq-1.0 \mathrm{~g} / \mathrm{dL}$. To evaluate the robustness of the primary analysis, supplementary analyses were performed (online suppl. Table 6). Analysis of the principal secondary endpoint was performed by using a logistic regression model including treatment, baseline hemoglobin, and prior ESA use to assess the superiority of daprodustat to CERA. Only when noninferiority was achieved would superiority be assessed to maintain an overall type I error rate of $2.5 \%$ (one-sided). 
Table 1. Baseline characteristics

\begin{tabular}{|c|c|c|}
\hline ITT population $^{\mathrm{a}}$ & $\begin{array}{l}\text { Daprodustat } \\
(n=108)\end{array}$ & $\begin{array}{l}\text { CERA } \\
(n=109)\end{array}$ \\
\hline Age, mean $( \pm S D)$, years & $69( \pm 11)$ & $71( \pm 9)$ \\
\hline \multicolumn{3}{|l|}{ Age group, $n(\%)$} \\
\hline$\leq 19$ years & $0(0)$ & $0(0)$ \\
\hline $20-64$ years & $30(28)$ & $16(15)$ \\
\hline$\geq 65$ years & $78(72)$ & $93(85)$ \\
\hline \multicolumn{3}{|l|}{ Sex, $n(\%)$} \\
\hline Male & $66(61)$ & $69(63)$ \\
\hline Female & $42(39)$ & $40(37)$ \\
\hline Weight, mean $( \pm S D), \mathrm{kg}$ & $59( \pm 11.2)$ & $61( \pm 11.9)$ \\
\hline BMI, mean $( \pm \mathrm{SD}), \mathrm{kg} / \mathrm{m}^{2}$ & $23( \pm 3.3)$ & $24( \pm 3.7)$ \\
\hline Hemoglobin, mean $( \pm S D), g / d L$ & $10.5( \pm 1.1)$ & $10.7( \pm 1.1)$ \\
\hline Ferritin, geometric mean (CV\%), $\mu \mathrm{g} / \mathrm{L}$ & $159(89)$ & $144(81)$ \\
\hline TSAT, mean $( \pm S D), \%$ & $32( \pm 11)$ & $32( \pm 12)$ \\
\hline Hepcidin, geometric mean (CV\%), ng/mL & $63(75)$ & $53(83)$ \\
\hline Oral iron use, ${ }^{\mathrm{b}} n(\%)$ & $30(28)$ & $29(27)$ \\
\hline \multicolumn{3}{|l|}{ CKD stage, $n(\%)$} \\
\hline G3 & $8(7)$ & $17(16)$ \\
\hline G4 & $54(50)$ & $48(44)$ \\
\hline G5 & $46(43)$ & $44(40)$ \\
\hline Prior ESA user, $n(\%)$ & $58(54)$ & $59(54)$ \\
\hline \multicolumn{3}{|l|}{ Prior ESA type, $n(\%)$} \\
\hline Epoetin & $0(0)$ & $0(0)$ \\
\hline CERA & $33(31)$ & $38(35)$ \\
\hline Darbepoetin alfa & $25(23)$ & $21(19)$ \\
\hline \multicolumn{3}{|l|}{ Prior ESA dose, mean $( \pm S D)$} \\
\hline Epoetin, IU/week & na & na \\
\hline CERA, $\mu \mathrm{g} / 4$ weeks & $72.4( \pm 38.84)$ & $65.2( \pm 36.16)$ \\
\hline Darbepoetin alfa, $\mu \mathrm{g} / 4$ weeks & $64.0( \pm 37.08)$ & $66.4( \pm 40.92)$ \\
\hline Safety population ${ }^{c}$ & $\begin{array}{l}\text { Daprodustat } \\
(n=149)\end{array}$ & $\begin{array}{l}\text { CERA } \\
(n=150)\end{array}$ \\
\hline Age, mean $( \pm S D)$, years & $68( \pm 12)$ & $70( \pm 9)$ \\
\hline \multicolumn{3}{|l|}{ Age group, $n(\%)$} \\
\hline$\leq 19$ years & $0(0)$ & $0(0)$ \\
\hline 20-64 years & $45(30)$ & $24(16)$ \\
\hline$\geq 65$ years & $104(70)$ & $126(84)$ \\
\hline \multicolumn{3}{|l|}{ Sex, $n(\%)$} \\
\hline Male & $96(64)$ & $92(61)$ \\
\hline Female & $53(36)$ & $58(39)$ \\
\hline Weight, mean $( \pm S D), \mathrm{kg}$ & $60( \pm 11.8)$ & $62( \pm 13.6)$ \\
\hline BMI, mean $( \pm S D), \mathrm{kg} / \mathrm{m}^{2}$ & $23( \pm 3.4)$ & $24( \pm 4.2)$ \\
\hline \multicolumn{3}{|l|}{ CKD stage, $n(\%)$} \\
\hline G3 & $15(10)$ & $20(13)$ \\
\hline G4 & $74(50)$ & $63(42)$ \\
\hline G5 & $60(40)$ & $67(45)$ \\
\hline Prior ESA user, $n(\%)$ & $58(39)$ & $59(39)$ \\
\hline \multicolumn{3}{|l|}{ Prior ESA type, $n(\%)$} \\
\hline Epoetin & $0(0)$ & $0(0)$ \\
\hline CERA & $33(22)$ & $38(25)$ \\
\hline Darbepoetin alfa & $25(17)$ & $21(14)$ \\
\hline
\end{tabular}


Table 1 (continued)

\begin{tabular}{|c|c|c|}
\hline Safety population ${ }^{c}$ & $\begin{array}{l}\text { Daprodustat } \\
(n=149)\end{array}$ & $\begin{array}{l}\text { CERA } \\
(n=150)\end{array}$ \\
\hline \multicolumn{3}{|l|}{ Prior ESA dose, mean $( \pm S D)$} \\
\hline Epoetin, IU/week & na & na \\
\hline CERA, $\mu \mathrm{g} / 4$ weeks & $72.4( \pm 38.84)$ & $65.2( \pm 36.16)$ \\
\hline Darbepoetin alfa, $\mu \mathrm{g} / 4$ weeks & $64.0( \pm 37.08)$ & $66.4( \pm 40.92)$ \\
\hline Hypertension, $n(\%)$ & $141(95)$ & $145(97)$ \\
\hline Diabetes, $n(\%)$ & $65(44)$ & $69(46)$ \\
\hline Hyperlipidemia, $n(\%)$ & $91(61)$ & $105(70)$ \\
\hline Angina pectoris, $n(\%)$ & $12(8)$ & $10(7)$ \\
\hline Diabetic retinopathy, $n(\%)$ & $42(28)$ & $39(26)$ \\
\hline Macular edema, $n(\%)$ & $8(5)$ & $5(3)$ \\
\hline Age-related macular degeneration, $n(\%)$ & $4(3)$ & $4(3)$ \\
\hline \multicolumn{3}{|c|}{ 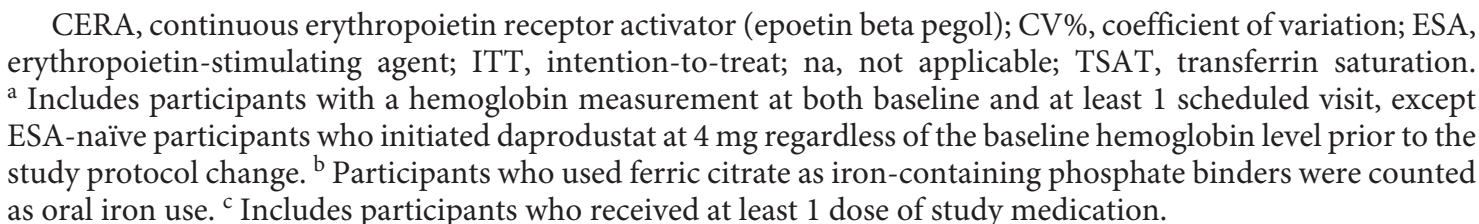 } \\
\hline
\end{tabular}

The number and percentage of AEs were summarized by primary system organ class and MedDRA preferred terms, version 21.1. No interim analysis was planned. Statistical analyses were performed with SAS (version 9.4; Cary, NC, USA).

\section{Results}

\section{Participant Disposition and Baseline Characteristics}

A total of 299 patients (149 in the daprodustat group, 150 in the CERA group) were randomized, of whom 217 were included in the ITT population (100 ESA-naïve, 117 ESA users; Fig. 1); 111 participants (74\%) in the daprodustat group and $113(75 \%)$ in the CERA group completed the study. The median duration of exposure was 365 days in both treatment groups (range: 6-372 daprodustat, 56370 CERA).

Baseline characteristics of the ITT population were generally balanced between groups (Table 1). The percentage of participants aged $\geq 65$ years was lower in the daprodustat group (72\%) than in the CERA group (85\%). Severity of CKD was slightly greater in the daprodustat group (CKD stage $\mathrm{G} 3,7 \%$; stage $\mathrm{G} 5,43 \%$ ) than in the CERA group (CKD stage G3, 16\%; stage G5, 40\%).

\section{Hemoglobin Levels}

The point estimates for mean hemoglobin level during the primary efficacy evaluation period were $12.0 \mathrm{~g} / \mathrm{dL}$
(95\% CI 11.8-12.1) for daprodustat and 11.9 (95\% CI 11.7-12.0) for CERA with a treatment difference of 0.1 ( $95 \%$ CI -0.1 to 0.3 ). Lower limits of the $95 \%$ CI for the difference between treatment groups were greater than the prespecified margin $(-1.0 \mathrm{~g} / \mathrm{dL})$. Subgroup analyses by prior ESA were consistent with the overall population (online suppl. Table 7). Supplementary analyses to evaluate the robustness of the primary efficacy result are shown in online suppl. Table 6.

In the modified ITT population, 92\% (daprodustat 81/88, CERA 80/87) of the participants in each treatment group had a mean hemoglobin level within the target range during the primary efficacy evaluation period (odds ratio daprodustat vs. CERA, 1.01; 95\% CI 0.33-3.04). Subgroup analyses by prior ESA were consistent with the overall population (online suppl. Table 7).

Overall, mean hemoglobin levels reached the target range at week 8 in both treatment groups and were maintained within the target range until week 52. For ESAnaïve participants, mean hemoglobin levels reached the target range at week 8 in both treatment groups and were maintained until week 52. For ESA users, mean hemoglobin levels were maintained within the target range throughout the treatment period in both treatment groups (Fig. 2).

Participants treated with daprodustat had hemoglobin levels within the target range during the primary efficacy evaluation period for 10.2 (SD 2.8) weeks compared with
30

Am J Nephrol 2021;52:26-35

DOI: $10.1159 / 000513103$
Nangaku et al. 
9.4 (SD 3.3) in the CERA group, a treatment difference of 0.8 (95\% CI -0.1 to 1.7$)$. ESA-naïve participants remained in the target range longer on daprodustat than on CERA (10.5 [2.6] weeks vs. 8.7 [3.6] weeks), a treatment difference of 1.8 (95\% CI 0.4-3.2). In ESA users, the mean duration was similar between treatment groups (online suppl. Table 8).

For ESA-naïve participants, the mean hemoglobin level change from baseline at week 4 was $0.8 \mathrm{~g} / \mathrm{dL}$ (SD 0.6) in the daprodustat group and $0.6(0.7) \mathrm{g} / \mathrm{dL}$ in the CERA group. Post hoc analysis showed the mean hemoglobin level change from baseline at week 4 was $0.7(0.5) \mathrm{g} / \mathrm{dL}$ for those with a 2 -mg starting dose $(n=44)$ and $1.1(0.9) \mathrm{g} /$ $\mathrm{dL}$ for participants with a 4 -mg starting dose $(n=6)$, respectively. Two of 44 participants in the $2-\mathrm{mg}$ group had an increase of $>1.0 \mathrm{~g} / \mathrm{dL}$ after 2 weeks and none increased $>2.0 \mathrm{~g} / \mathrm{dL}$ after 4 weeks. One of 6 patients with a starting dose of $4 \mathrm{mg}$ had an increase of $>2.0 \mathrm{~g} / \mathrm{dL}$ after 4 weeks. However, this participant took more daprodustat than instructed during the initial 2 weeks.

For ESA users, there was no clinically significant change in hemoglobin from baseline to week 4 (daprodustat, 0.2 [1.0] g/dL; CERA, 0.0 [0.7] g/dL). One of $58 \mathrm{pa}-$ tients in the daprodustat group had an increase in hemoglobin of $>2.0 \mathrm{~g} / \mathrm{dL}$, and 1 participant in each treatment group had a decrease in hemoglobin of at least $-2.0 \mathrm{~g} / \mathrm{dL}$ (these 3 participants had a baseline hemoglobin level of $\geq 11.0 \mathrm{~g} / \mathrm{dL})$.

\section{Daprodustat Dose}

The median (P25-P75) daprodustat doses during weeks 40-52 were 4.0 (2.3-6.0) $\mathrm{mg}$ /day in ESA-naïve and $5.3(3.3-7.3) \mathrm{mg} /$ day in ESA users. In CERA, the median (P25-P75) doses during weeks 40-52 were 91.7 (62.5133.3) $\mu \mathrm{g} / 4$ weeks in ESA-naïve patients and 91.7 (50.0 150.0) $\mu \mathrm{g} / 4$ weeks in ESA users (online suppl. Table 9). At week 48 , the majority of subjects were treated by daprodustat 4 and $6 \mathrm{mg}$ irrespective of baseline erythropoietin resistance index (ERI); however, the proportion of subjects treated with 12-, 18-, and 24-mg doses tended to be greater in the higher ERI ( $\geq 5.27 \mathrm{IU} / \mathrm{kg} /$ week $/ \mathrm{g} / \mathrm{dL})$ group (online suppl. Table 10).

Fig. 2. Mean hemoglobin level and 95\% CIs by study treatment group (ITT population). Overall ITT (a); ESA-naïve participants (b); ESA users (c). Light gray dashed lines indicate target hemoglobin range. Orange solid lines represent time courses of values in daprodustat users. CI, confidence interval; CERA, continuous erythropoietin receptor activator (epoetin beta pegol); ESA, erythropoiesis-stimulating agent; ITT, intention-to-treat population.

Daprodustat for Anemia in Japanese

Nondialysis Patients

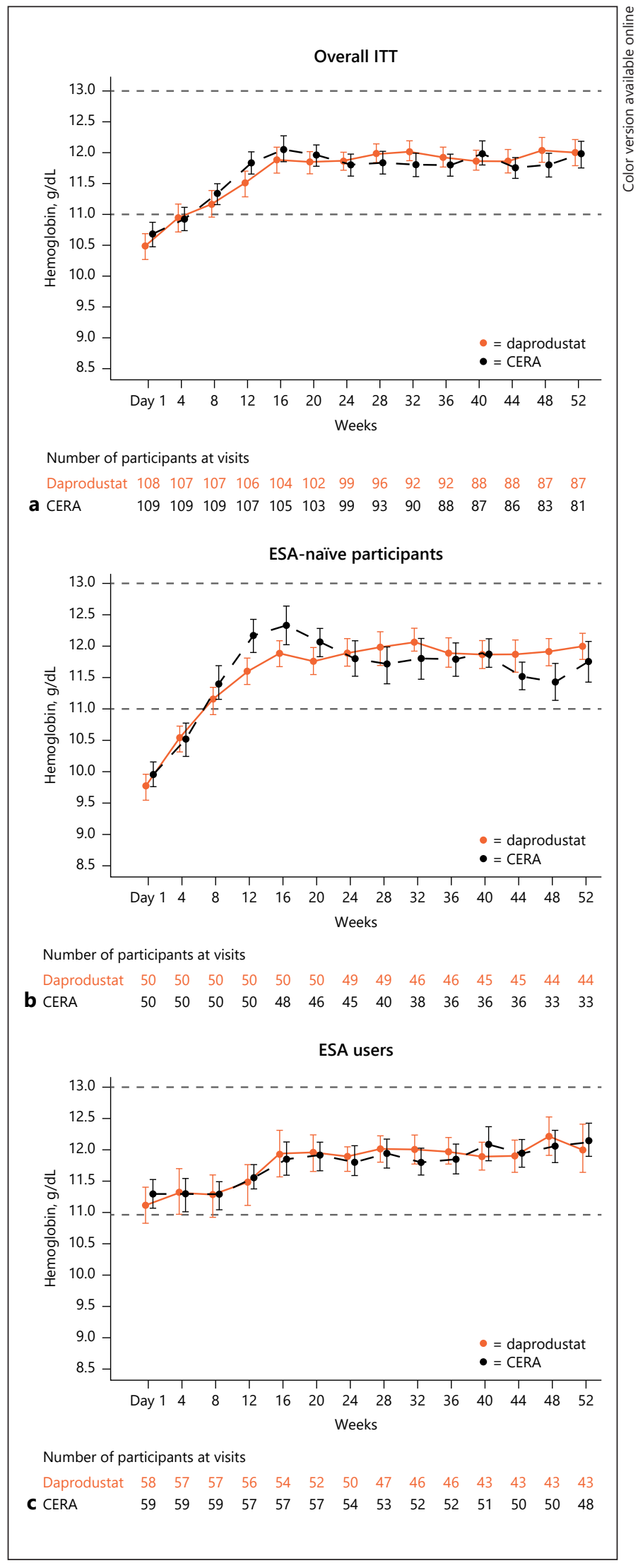

Am J Nephrol 2021;52:26-35

DOI: $10.1159 / 000513103$ 


\section{Iron Metabolism}

The percentage of participants using oral iron at baseline was similar between groups (daprodustat, 28\%; CERA, $27 \%$ ). Oral iron was used by $52 \%$ of participants in the daprodustat group during the overall treatment period, compared with $45 \%$ in the CERA group, respectively. One participant in the daprodustat group used intravenous iron. The monthly mean oral iron dose during the study was 1,615 (SD 1,251) $\mathrm{mg}$ in the daprodustat group and $1,662(1,151) \mathrm{mg}$ in the CERA group.

Baseline measures of iron status were similar for the two treatment groups (Table 1). At week 52, mean serum iron levels were unchanged in the daprodustat group and slightly increased from baseline during weeks $28-52$ for CERA, despite similar erythropoiesis (baseline: $87 \mu \mathrm{g} / \mathrm{dL}$ daprodustat, $83 \mu \mathrm{g} / \mathrm{dL}$ CERA; week 52: $90 \mu \mathrm{g} / \mathrm{dL}$ daprodustat, $92 \mu \mathrm{g} / \mathrm{dL}$ CERA) (Fig. 3a). Mean total iron-binding capacity levels increased from baseline throughout the treatment period for daprodustat but remained near baseline for CERA (baseline: $272 \mu \mathrm{g} / \mathrm{dL}$ daprodustat, 267 $\mu \mathrm{g} / \mathrm{dL}$ CERA; week 52: $316 \mu \mathrm{g} / \mathrm{dL}$ daprodustat, $268 \mu \mathrm{g} / \mathrm{dL}$ CERA) (Fig. 3b). The mean serum transferrin level was $2.0 \mathrm{~g} / \mathrm{L}$ at baseline in both treatment groups (safety population). At week 52, the mean transferrin level increased to $2.4 \mathrm{~g} / \mathrm{L}$ for daprodustat but remained near baseline for CERA. Mean TSAT levels decreased from baseline for daprodustat but increased by $10 \%$ in the CERA group (baseline: 32\% daprodustat, 32\% CERA; week 52: 29\% daprodustat, 34\% CERA) (Fig. 3c). Geometric mean ferritin levels decreased from baseline in both treatment groups (baseline: $159 \mu \mathrm{g} / \mathrm{L}$ daprodustat, $144 \mu \mathrm{g} / \mathrm{L}$ CERA; week 52: $106 \mu \mathrm{g} / \mathrm{L}$ daprodustat, $122 \mu \mathrm{g} / \mathrm{L}$ CERA) (Fig. 3d). At baseline, the geometric mean hepcidin level was $63 \mathrm{ng} /$ $\mathrm{mL}$ (95\% CI 55-71) for daprodustat and 53 (95\% CI 4661) for CERA. At week 52, there was a $45 \%$ reduction in hepcidin from baseline for daprodustat and a $10 \%$ increase for CERA (35 ng/mL daprodustat, $64 \mathrm{ng} / \mathrm{mL}$ CERA) (Fig. 3e).

\section{Safety}

During the 52-week treatment period, 92\% of participants taking daprodustat and $89 \%$ taking CERA experienced $\geq 1 \mathrm{AE}$ (Table 2$)$. The most common AEs ( $\geq 10 \%$ of participants in any group) were nasopharyngitis (33\% daprodustat, 37\% CERA) and constipation (7\% daprodustat, $12 \%$ CERA). Hyperkalemia was reported in $8 \%$ of patients in the daprodustat group versus 5\% in the CERA group.

Three fatal events occurred: 1 in the daprodustat group (shock hemorrhagic) and 2 in the CERA group (arrhythmia; aortic dissection). Serious AEs occurred in $23 \%$ of
Table 2. Summary of on-therapy AEs (reported in $\geq 5 \%$ of participants) (safety population ${ }^{\mathrm{a}}$ )

\begin{tabular}{lrr}
\hline AE & $\begin{array}{l}\text { Daprodustat } \\
(n=149)\end{array}$ & $\begin{array}{l}\text { CERA } \\
(n=150)\end{array}$ \\
\hline Any event, $n(\%)$ & $137(92)$ & $134(89)$ \\
Nasopharyngitis & $49(33)$ & $56(37)$ \\
Constipation & $10(7)$ & $18(12)$ \\
Back pain & $12(8)$ & $11(7)$ \\
Renal impairment & $9(6)$ & $13(9)$ \\
Hyperkalemia & $12(8)$ & $8(5)$ \\
Pruritus & $12(8)$ & $5(3)$ \\
CKD & $6(4)$ & $10(7)$ \\
Influenza & $8(5)$ & $8(5)$ \\
Contusion & $5(3)$ & $8(5)$ \\
Diarrhea & $5(3)$ & $7(5)$ \\
Blood pressure increased & $8(5)$ & $4(3)$ \\
Hypertension & $4(3)$ & $7(5)$ \\
Muscle spasms & $4(3)$ & $7(5)$ \\
\hline
\end{tabular}

AE, adverse event; CERA, continuous erythropoietin receptor activator (epoetin beta pegol). ${ }^{\text {a }}$ The safety population included all participants who received at least 1 dose of study medication.

patients on daprodustat and $29 \%$ on CERA. Serious AEs reported in $\geq 3$ participants (2\%) per treatment group were renal impairment (3\% daprodustat, 7\% CERA), CKD (reported by investigators as worsening/progression of CKD; $4 \%, 5 \%)$, ESRD (2\%, $0 \%)$, and heart failure congestive $(0 \%, 3 \%)$. In $9 \%$ of patients in both treatment groups, AEs led to discontinuation of the study drug.

There were no meaningful differences in AEs of special interest between treatment groups. Cancers occurred in $2 \%$ of patients in both treatment groups, and ocular AEs of special interest occurred in 3\% of patients in both treatment groups (online suppl. Table 11). There was little or no mean change from baseline in blood pressure within and between treatment groups. Fewer participants taking daprodustat had a change in antihypertensive medications due to increased blood pressure than those taking CERA (38 vs. $45 \%$, respectively) (online suppl. Table 12 ).

\section{Clinical Laboratory Parameters}

Small decreases in mean total cholesterol, HDL cholesterol, and LDL cholesterol levels were observed in the daprodustat group, while an increase in HDL cholesterol and little change in total and LDL cholesterol were observed in the CERA group (online suppl. Fig. 1). Elevated potassium $(>6.0 \mathrm{mmol} / \mathrm{L})$ was reported in $7 \%$ for daprodustat versus $10 \%$ for CERA.
Nangaku et al. 


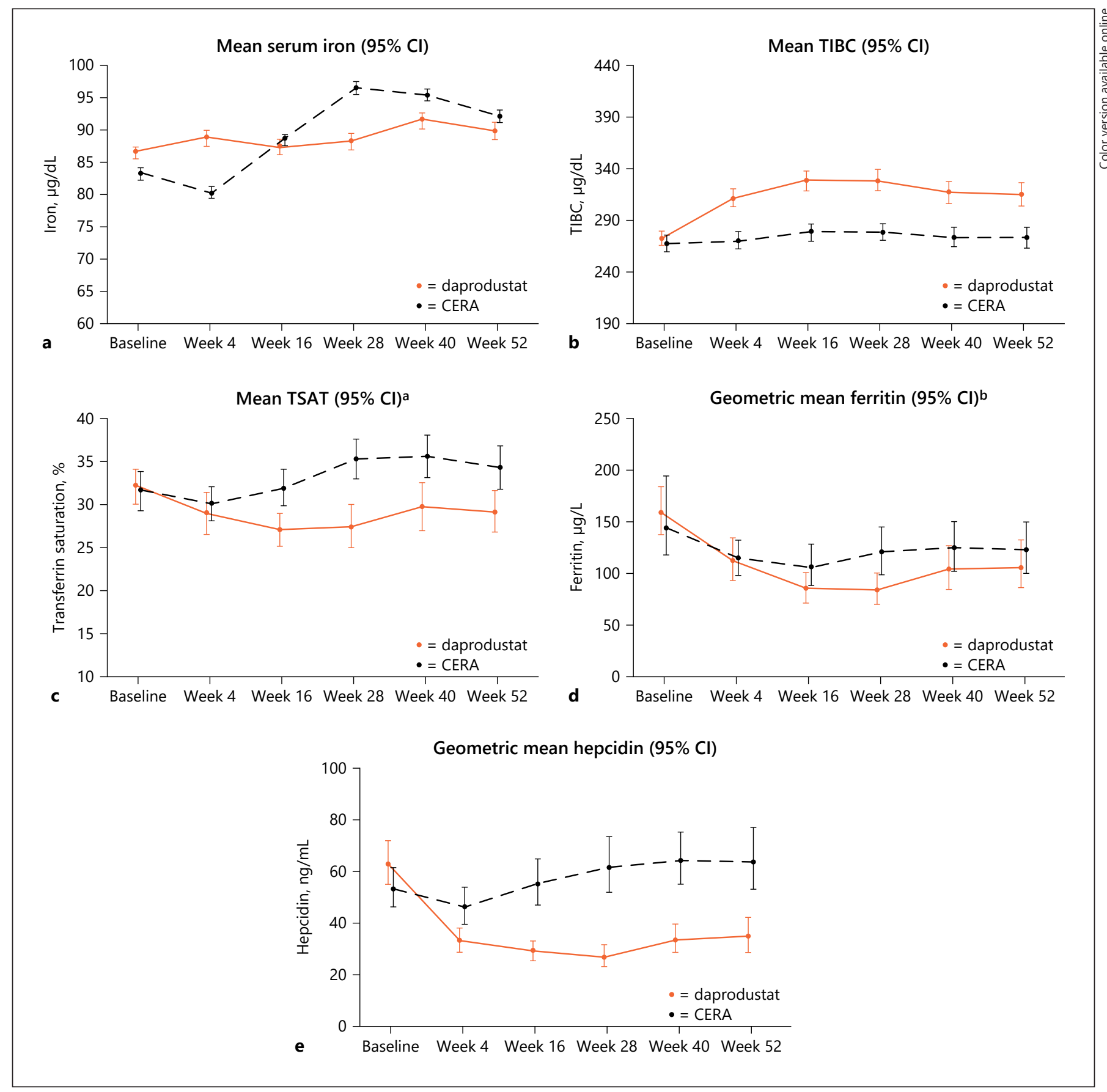

Fig. 3. Effect of daprodustat on iron metabolism (ITT population). Mean serum iron level (95\% CI) (a); mean TIBC level (95\% CI) (b); mean TSAT level (95\% CI) (c); geometric mean ferritin level (95\% CI) (d); geometric mean hepcidin level (95\% CI) (e). Orange solid lines represent time courses of values in daprodustat users.

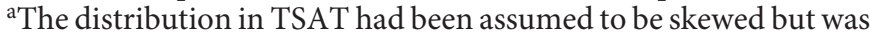
found not to be skewed after the data review; therefore, the post hoc analysis of TSAT was not based on transformed values. ${ }^{\mathrm{b}}$ The distributions were skewed and required a log transformation for the analysis. The distribution in ferritin had been assumed not to be skewed but was found to be skewed after the data review; therefore, the analysis of ferritin based on a log transformation was done as a post hoc analysis. CERA, continuous erythropoietin receptor activator (epoetin beta pegol); CI, confidence interval; ITT, intention-to-treat; TIBC, total iron-binding capacity; TSAT, transferrin saturation. 


\section{Discussion}

In this first year-long study of the oral HIF-PHI, daprodustat, in ND patients with $\mathrm{CKD}$, daprodustat was noninferior to CERA for achieving and maintaining target hemoglobin while avoiding the need for regular injections. In both ESA-naïve participants and ESA users, daprodustat effectively provided consistent and durable control of hemoglobin. In ESA-naïve participants enrolled after the protocol amendment, daprodustat increased hemoglobin in a controlled manner, with few patients experiencing a rapid rise in hemoglobin $(>2 \mathrm{~g} / \mathrm{dL}$ per 4 weeks), when started at $2 \mathrm{mg}$ with baseline hemoglobin levels $\geq 9.0 \mathrm{~g} / \mathrm{dL}$ and at $4 \mathrm{mg}$ with baseline hemoglobin levels $<9.0 \mathrm{~g} / \mathrm{dL}$. Mean hemoglobin levels reached the target range by week 8 in both treatment groups. The effect of daprodustat was durable up to 52 weeks, and in this study, ESA-naïve participants remained in the target range longer during weeks $40-52$ than patients treated with CERA. In ESA users, switching to daprodustat maintained hemoglobin with only a few participants experiencing a rapid rise or major decrease, and mean hemoglobin levels remained within the target range throughout 1 year. The duration ESA users remained in the target range during weeks $40-52$ was similar to that of participants treated with CERA.

Daprodustat was associated with decreases in hepcidin, whereas CERA was not, despite matched hemoglobin in the two treatment groups. Thus, the decrease in hepcidin with daprodustat treatment, accompanied by decreases in ferritin, may result from a shift from iron storage to erythrocyte development compared with CERA. Observed increases in total iron-binding capacity were likely determined by transferrin, which is increased by HIF $[10,17]$. A larger percentage of participants in the daprodustat group received oral iron; however, the monthly oral iron dose was low. In addition, the decision to start oral iron supplementation was at the discretion of the investigator, based on iron supplement criteria. Therefore, it is not clear if iron availability differed between the treatment groups in this study.

Daprodustat was generally well tolerated in Japanese ND patients with an AE profile comparable to that of CERA. The safety profile for daprodustat in this study was similar to prior international phase 2 studies $[9,14]$ and Japanese phase 3 studies [18]. This study is of insufficient size and duration to evaluate cardiovascular events. However, 2 large studies designed to assess the effect of daprodustat on cardiovascular risk are ongoing (NCT02879305 and NCT02876835).
Ophthalmologic examinations were performed because of concern that increases in vascular endothelial growth factor, which might be increased via increased HIF, could exacerbate proliferative retinopathy and macular edema [10]. In our study, there were no eye-related exclusion criteria, which resulted in a quarter of patients entering the study with diabetic retinopathy. No difference between treatment groups in ocular AEs of special interest was observed during 1 year of treatment in this population of patients, consistent with previous findings suggesting daprodustat does not exacerbate these conditions $[9,14,18]$.

Hyperkalemia was reported in a phase 3 study for another HIF-PHI, roxadustat, in Chinese ND CKD patients (16\% in the roxadustat group vs. $8 \%$ for placebo) [19]. There is no obvious mechanism for HIF-linked interference with potassium homeostasis. In our study, elevated potassium $(>6.0 \mathrm{mmol} / \mathrm{L})$ was reported in $7 \%$ in the daprodustat group versus $10 \%$ with CERA, and AEs of hyperkalemia were reported in $8 \%$ with daprodustat versus 5\% with CERA. These data do not support an effect of daprodustat on potassium levels. While statin use during the treatment period was generally balanced between groups, small changes in lipid parameters were noted with daprodustat in the current study as reported previously with roxadustat [19]. However, the underlying mechanism is unclear.

The study's 1-year duration limits the assessment of long-term risk with daprodustat. Additionally, the openlabel design may be subject to AE reporting biases and preferential reporting of events described in the investigator brochure for a novel treatment. This is less likely to have affected more objective measures such as the efficacy assessment using hemoglobin.

\section{Conclusions}

In conclusion, oral daprodustat was noninferior to CERA for treatment of anemia in Japanese ND patients with CKD. Daprodustat was generally well tolerated, with an AE profile similar to comparator ESA treatment with no new safety concerns identified.

\section{Acknowledgements}

Editorial support (assembling tables and figures, collating author comments, copyediting, fact checking, and referencing) was provided by Allyson Lehrman, DPM, and graphic services were provided by AOIC, LLC, and were funded by GSK.
Nangaku et al. 


\section{Statement of Ethics}

This study was approved by the Institutional Review Board (online suppl. Table 1) at every participating institution and was conducted according to the recommendations of Good Clinical Practice and the Declaration of Helsinki. All participants provided written, informed consent to participate in the study.

\section{Conflict of Interest Statement}

M.N. has received grants and personal fees from Astellas Pharma Inc. (Astellas), Chugai Pharmaceutical Co. Ltd. (Chugai), Daiichi Sankyo Co. Ltd., GlaxoSmithKline (GSK), Kyowa Kirin Co. Ltd. (KKC), Mitsubishi Tanabe Pharma, and Torii Pharmaceutical Co. Ltd. (Torii); grants from Bayer Yakuhin Ltd. (Bayer), Ono Pharmaceutical Co. Ltd. (Ono), and Takeda Pharmaceutical Co. Ltd. (Takeda); and personal fees from AstraZeneca and JT Pharmaceuticals. T.H. has received grants and personal fees from Asahi Kasei Pharma Corporation, Bayer, Chugai, Kissei Pharmaceutical Co. Ltd. (Kissei), Otsuka Pharmaceutical Co. Ltd. (Otsuka), and Torii; grants, personal fees, and other from KKC, and Ono; personal fees and other from Astellas; grants from Eisai Co. Ltd., Fuso Pharmaceutical Industries Ltd. (Fuso), Takeda, and Terumo Corporation; and other from GSK. T.A. has received personal fees from Astellas, Bayer, Chugai, Fuso, GSK, JT Pharmaceuticals, KKC, Kissei, Nipro Corporation, Ono, Otsuka, Torii, and Sanwa Chemical Industrial Co. Ltd. Y.T. has received personal fees from Chugai, GSK, KKC, and Torii. R.N. is an employee of GSK. N.O., K.K., T.N., N.P.J., Y.E., and A.R.C. are employees of and hold equity stock in GSK.

\section{Funding Sources}

Funding for this study (NCT02791763, available from www. clinicaltrials.gov) was provided by GlaxoSmithKline (GSK).

\section{Author Contributions}

All listed authors meet the criteria for authorship set forth by the International Committee for Medical Journal Editors. M.N., T.H., T.A., Y.T., Y.E., and A.R.C. contributed to the concept and design of the study. All authors contributed to the data analysis or interpretation. All authors contributed to critical revision and final approval of the manuscript. All authors agree to take responsibility for the manuscript's content.

\section{Availability of Data and Material}

Within 6 months of this publication, anonymized individual participant data, the annotated case report form, protocol, reporting and analysis plan, dataset specifications, raw dataset, analysisready dataset, and clinical study report will be available for research proposals approved by an independent review committee. Proposals should be submitted to www.clinicalstudydatarequest. com. A data access agreement will be required.

\section{References}

1 Akizawa T, Makino H, Matsuo S, Watanabe T, Imai E, Nitta K, et al. Management of anemia in chronic kidney disease patients: baseline findings from chronic kidney disease Japan cohort study. Clin Exp Nephrol. 2011 Apr;15(2):248-57.

2 Yamamoto H, Nishi S, Tomo T, Masakane I, Saito K, Nangaku M, et al. 2015 Japanese society for dialysis therapy: guidelines for renal anemia in chronic kidney disease. Ren Replace Ther. 2017 Jun;3(1):1-46.

3 Aranesp [package insert]. Thousand Oaks, CA: Amgen Inc.; 2019 Jan. Available from: https:// www.pi.amgen.com/ /media/amgen/repositorysites/pi-amgen-com/aranesp/ckd/aranesp_pi_hcp_english.pdf. Accessed 2019 Apr 4.

4 Pfeffer MA, Burdmann EA, Chen CY, Cooper ME, de Zeeuw D, Eckardt KU, et al. Baseline characteristics in the trial to reduce cardiovascular events with aranesp therapy (TREAT). Am J Kidney Dis. 2009 Jul;54(1):59-69.

5 Besarab A, Bolton WK, Browne JK, Egrie JC, Nissenson AR, Okamoto DM, et al. The effects of normal as compared with low hematocrit values in patients with cardiac disease who are receiving hemodialysis and epoetin. N Engl J Med. 1998 Aug;339(9):584-90.

6 Drueke TB, Locatelli F, Clyne N, Eckardt KU, Macdougall IC, Tsakiris D, et al. Normalization of hemoglobin level in patients with chronic kidney disease and anemia. N Engl J Med. 2006 Nov;355(20):2071-84.

7 Procrit [package insert]. Thousand Oaks, CA: Amgen Inc.; 2008.

8 Singh AK, Szczech L, Tang KL, Barnhart H, Sapp S, Wolfson M, et al. Correction of anemia with epoetin alfa in chronic kidney disease. $\mathrm{N}$ Engl J Med. 2006 Nov;355(20):2085-98.

9 Holdstock L, Cizman B, Meadowcroft AM, Biswas N, Johnson BM, Jones D, et al. Daprodustat for anemia: a 24-week, open-label, randomized controlled trial in participants with chronic kidney disease. Clin Kidney J. 2019 Feb;12(1):129-38.

10 Lenihan CR, Winkelmayer WC. The dawning of a new day in CKD anemia care? J Am Soc Nephrol. 2016 Apr;27(4):968-70.

11 Hasegawa S, Tanaka T, Nangaku M. Hypoxiainducible factor stabilizers for treating anemia of chronic kidney disease. Curr Opin Nephrol Hypertens. 2018 Sep;27(5):331-8.

12 Sakashita M, Tanaka T, Nangaku M. Hypoxiainducible factor-prolyl hydroxylase domain inhibitors to treat anemia in chronic kidney disease. Contrib Nephrol. 2019;198:112-23.

13 Sugahara M, Tanaka T, Nangaku M. Prolyl hydroxylase domain inhibitors as a novel therapeutic approach against anemia in chronic kidney disease. Kidney Int. 2017 Aug; 92(2):306-12.
14 Meadowcroft AM, Cizman B, Holdstock L, Biswas N, Johnson BM, Jones D, et al. Daprodustat for anemia: a 24-week, open-label, randomized controlled trial in participants on hemodialysis. Clin Kidney J. 2019 Feb;12(1): 139-48.

15 Akizawa T, Tsubakihara Y, Nangaku M, Endo Y, Nakajima H, Kohno T, et al. Effects of daprodustat, a novel hypoxia-inducible factor prolyl hydroxylase inhibitor on anemia management in Japanese hemodialysis subjects. Am J Nephrol. 2017;45(2):127-35.

16 Tsubakihara Y, Nishi S, Akiba T, Hirakata H, Iseki K, Kubota M, et al. 2008 Japanese society for dialysis therapy: guidelines for renal anemia in chronic kidney disease. Ther Apher Dial. 2010 Jun;14(3):240-75.

17 Rolfs A, Kvietikova I, Gassmann M, Wenger RH. Oxygen-regulated transferrin expression is mediated by hypoxia-inducible factor-1. J Biol Chem. 1997;272(32):20055-62.

18 Tsubakihara Y, Akizawa T, Nangaku M, Onoue T, Yonekawa T, Matsushita H, et al. A 24week anemia correction study of daprodustat in Japanese dialysis patients. Ther Apher Dial. 2020 Apr;24(2):108-14.

19 Chen N, Hao C, Peng X, Lin H, Yin A, Hao L, et al. Roxadustat for anemia in patients with kidney disease not receiving dialysis. N Engl J Med. 2019 Sep;381(11):1001-10. 